\title{
ON THE STABILITY OF THE TRACK OF THE SPACE ELEVATOR
}

\author{
Nicola Pugno \\ Professor \\ Department of Structural Engineering \\ Politecnico di Torino \\ Torino (Italy) \\ nicola.pugno@polito.it \\ Hans Troger, Alois Steindl, Michael Schwarzbart \\ Institute of Mechanics and Mechatronics \\ Vienna University of Technology \\ Vienna (Austria)
}

Hans.Troger@tuwien.ac.at, Alois Steindl@tuwien.ac.at, Michael.Schwarzbart@tuwien.ac.at

\begin{abstract}
A string moving with geostationary angular velocity in its radial relative equilibrium configuration around the Earth, reaching from the surface of the Earth far beyond the geostationary height, could be used as track for an Earth to space elevator. This is an old dream of mankind, originating about 100 years ago in Russia. Besides the question of feasibility from a technological point of view also the question concerning the stability of such a configuration has not yet been completely solved. Under the assumption that a proper material (defective carbon nanotubes) is available, making the connection possible technologically, we address the question of stability of the radial relative equilibrium of a tapered string on a circular geosynchronous orbit around the Earth, reaching from the surface of the Earth far beyond the geostationary height.
\end{abstract}

\section{Introduction}

One of the main problems of modern space exploration and space technology is the high cost of sending a payload from the surface of the Earth into space. Depending on the destination in space in the year 2000 these costs were about $10^{4}-10^{6}$ US Dollars for one kilogram of payload, because in order to carry the payload, rockets have to move a multiple of the amount of the payload due to their own weight and the necessary fuel. Hence for a long time there have been other ideas around for a cheaper way of transporting payloads into a space orbit. The most promising was proposed in 1960 by Artsutanov [1] to build a celestial elevator from the surface of the Earth to a satellite in geostationary orbit by hanging down a string from the satellite to the surface of the Earth. Such a string in its radial configuration could be used as track for a space elevator to provide easy access to a space orbit. This idea was investigated for its feasibility by Isaacs et al. 1966 ([2]) for a string with constant cross-section and by Pearson 1975 ([3]) for a weight- optimized string with tapered cross section. Both investigations came to the conclusion that at the time of the investigation no material was available to realize such a connection. Artsutanov's idea is

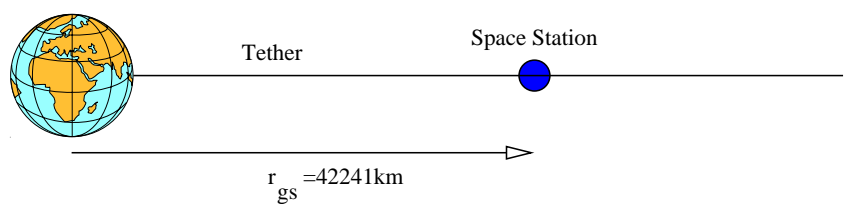

Figure 1: String connecting a satellite in geostationary orbit with the surface of the Earth. As counterweight another string must be deployed outside the geostationary height. For a minimum weight design its length is given in Fig. 4

based on the fact that a massive string moving on a circular orbit around the Earth, under the action of gravitational and centrifugal accelerations, finally will reach a relative equilibrium position, which is its streched radial position. In this equilibrium position the string is under tension (Beletsky and Levin [4]). To explain this we note that the motion 
center, which is defined by the equality of centrifugal and gravitational accelerations, is located at the geostationary orbit (one revolution/day). The motion centre is different from the centre of mass and the centre of gravity of the string. For a mass element of the string located below the geostationary radius the value of the gravitational acceleration acting on it is larger than the value of the corresponding centrifugal acceleration and for a mass element above the geostationary radius the value of the centrifugal acceleration is larger than that of the gravitational acceleration. Thus below the geostationary height the net force acting at a string element is pointing towards the Earth and above the geostationary height the net force is pointing away from the Earth. Decomposing these forces into a component in the direction of the straight line connecting these two elements and perpendicular to this line, results that the string is under tension and further a moment is created turning the string into the radial direction, as it is depicted for a dumb-bell satellite, which is a system of two point masses connected by a massless rigid rod, in Fig.2. This intuitive reasoning convinces some scientists that the radial configuration is stable. In order to

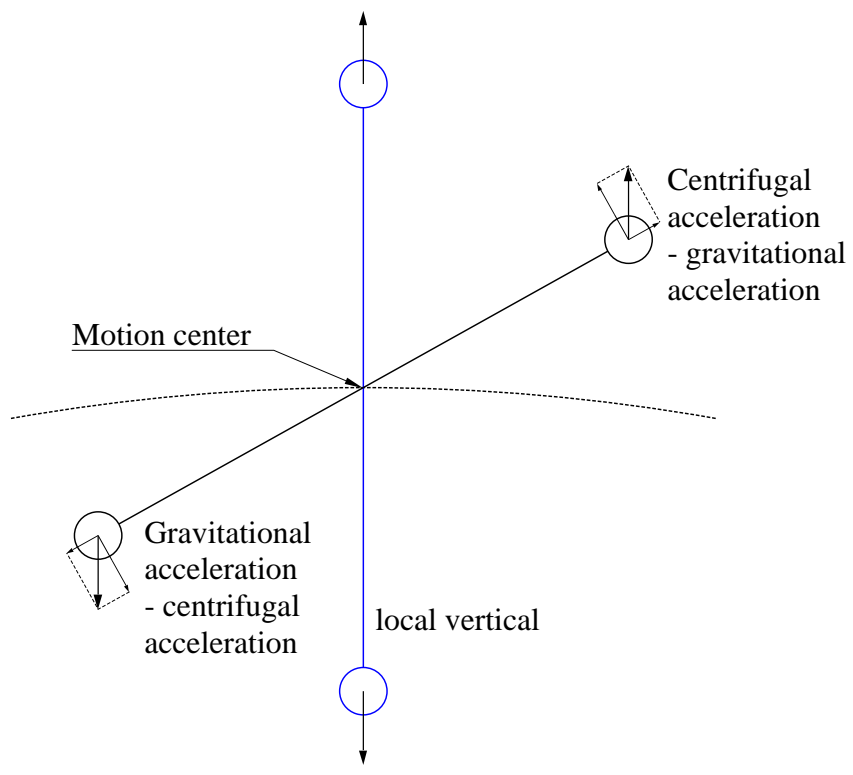

Figure 2: Dumbell-satellite consisting of two point masses connected by a massless rigid rod. Its motion centre moves on the geostationary orbit.

obtain a minimum-weight design the shape of the string must be tapered, such that in each crosssection the maximum admissible stress is reached.
This results in a shape, where the string is thickest at the point of highest tension, which is at the geosynchronous radius, and thinnest where the tension is lowest, namely at its ends (Fig. (4).

Of course, one has to compensate for the weight of the tapered string hanging down. If this is done by another string extending outward from the geostationary radius $(35863 \mathrm{~km}$ altitude above the surface of the Earth (Fig.11)), the length of this string must be several times the length to the geostationary orbit (Edwards [5]), if this part of the string is also designed for minimum weight (see also Fig. 4). From a technical point of view, placing a counterweight at the far end of a shorter string, once the inner end is fixed, could simplify the construction and will also result in a radial configuration reaching from the surface of the Earth moving with geostationary angular velocity. However, extending the string beyond the geostationary height is very important for the application as satellite launcher, because if a payload is placed on the string in a position farther away from the Earth than the geosynchronous height, simply by separating the payload from the string, it can be launched into a larger elliptical orbit or even out of the Earth gravitational field to perform interplanetary missions (Pearson 3]).

Until 1991 all these ideas were purely academic, since no material was available to realize such a project. However, at that time socalled "carbon nanotubes" were discovered, which are Fullerenes, that is cylindrical macromolecules composed of carbon atoms which are formed from a flat periodical hexagonal lattice with the thickness of the size of an atom (Ruoff et al [6], Pantano et al. [7]). Single walled nanotubes have been produced with a diameter of a few Nanometers $\left(1[\mathrm{~nm}]=10^{-9}[\mathrm{~m}]\right)$ and a length of the order of Centimeters. Hence so far an aspect ratio of order $10^{7}$ has been reached. Single walled nanotubes form the building block of multi walled nanotubes. Moreover, it is conceived to have bundels of nanotubes, from which one can expect to form nanoropes or nanosheets by a technological process similar to that of weaving a textile. For the perfect nanotubes such a structure may have a theoretical strength 100 times higher than steel, but with only one-sixth of the weight of steel. Moreover, besides their extreme strength, carbon nanotubes also allow large strains up to 16$24 \%$. 
The ratio between tensile strength and density is crucial for the taper ratio of the string, that is, the ratio between the area of the cross section of the string at the geosynchronous orbit to the area of the cross section at the surface of the Earth. For example, from the calculations performed in Edwards [5] the taper ratio required for steel would be $1.7 \times 10^{33}$, for Kevlar $2.6 \times 10^{8}$ and for carbon nanotubes 1.5. Our calculations which have been performed for endmasses on each side of the string of $1 \mathrm{~kg}$, resulted in ratios between the crosssectional area at the geostationary height to the area at the surface of the Earth, as can be seen from Fig.4, of 1.35 for $\sigma_{c}=150 \mathrm{GPa}$ and 2.50 for $\sigma_{c}=65 \mathrm{GPa}$. The second value uses the strength, which has been measured in experiments, since the theoretical strength cannot be expected to hold for a technical realization. The reason is that defects in the atomic lattice result in a reduction of the strength and also of Young's modulus. However there are no data reported concerning the decrease of the value of the Young's modulus. Hence we include a section, where we investigate what can be realistically expected concerning strength and elasticity modulus in a technical realization.

A careful, practically relevant investigation of the stability of the system's relative equilibrium would have to take into account various perturbations, such as the gravitational attraction of the Moon, atmospheric drag and payloads moving up and down the string. These effects and others have been addressed in Isaacs et al [2] and Pearson [3]. Interestingly enough the simpler question of stability of the radial relative equilibrium position of a long unperturbed string in the spherical symmetric Newtonian gravitational field has not been given much attention. However, this stability cannot be taken for granted in any case, because in Beletsky and Levin [4] and Krupa et al. [8] it is shown that a dumbell satellite possesses a stable radial relative equilibrium position only if the distance between the two masses is significantly smaller than the radius of the orbit. This loss of stability is an orbital instability (Krupa et al. [8]), which means that perturbations of the orbital radius grow, whereas perturbations of the attitude are still stable. It is caused by the strong nonlinearity of the gravitational field close to the center of the orbit.

In [9] it is shown that a pure continuous massive string, which may form the track of the space elevator, does not have a stable radial relative equilibrium position. This does not have significant consequences for the track since adding a satellite at the geostationary height, which practically will be the case, will have a stabilizing effect. Hence we also calculate the mass of a satellite at the geostationary height, which would be necessary to stabilize the radial configuration.

The calculations performed in [9] are improved, especially for the data, which we have calculated in Section 2, The stability of the relative equilibrium is evaluated by the Reduced Energy Momentum Method (REMM) (see Lewis and Simo [10, Marsden et al.[11], Marsden [12], Marsden and Ratiu [13]). The REMM is a generalisation of Routh's method as presented for example in Karapetyan and Rumyantsev [14]. We note that for cases considered in this paper, where cyclic coordinates are present, the REMM reduces to Routh's method. However, the REMM can be also applied if noncyclic coordinates are used. Applications of the REMM to tethered satellite systems are given in Wang et al [15] and Krupa et al [16].

\section{Carbon Nanotubes}

Almost in all calculations concerning thr Space elevator the theoretical strength and material data of Carbon Nanotubes has been used. However like any industrial product they will be imperfect and hence it is interesting to see what the influence on the taper ratio of the string would be if the string is made from imperfect carbon nanotubes.

We give a short fracture mechanics calculation, how the strength and Young's modulus may be changed if defective carbon nanotubes are used. More details can be found in the indicated literature.

\subsection{Young's modulus}

- We consider a single nanotube having thickness $t$, radius $r$ and length $l$, under tension $\sigma$ (or force $F=2 \pi r t \sigma$ ) and containing a nanocrack of length $2 a$ orthogonal (most critical configuration) to the applied load. The variation of the total potential energy induced by the presence of the crack 
is $\Delta W=\Delta L-F \Delta \delta$, where $L$ is the elastic energy stored in the nanotube and $\delta=F / S$ is the elastic displacement; $S$ is thus the nanotube's stiffness, i.e., $S=2 \pi r t E / l$, with $E$ Young's modulus. Applying Clapeyron's Theorem (see [17]) $\Delta L=F \Delta \delta / 2$ and consequently

$$
\Delta W=\frac{F^{2} \Delta S}{2 S^{2}}
$$

(the same result can be deduced for imposing the displacement rather than imposing the force). Furthermore, according to fracture mechanics $d W=$ $-G d A$, where $A$ is the crack surface area, i.e. in our case, $A=2 a t$, and $G$ is the energy release rate (see [17]). According to fracture mechanics the crack will propagate when $G$ reaches a critical value $G_{C}$, the so-called material fracture energy (per unit area). The energy release rate is related to the stress-intensity factor $K$ at the tip of the crack (derivable for different configurations from the stress-intensity factor Handbooks) via Irwin's correlation (see [17]) $G=K^{2} / E$. Let us consider the presence of an isolated crack. For simplicity we neglect the energy associated with the nanotube's circumferential curvature as well as the crack tip's self-interactions. Then $K=\sigma \sqrt{\pi a}$, since this case is analogous to the well-know Griffith's case (see [17]). Consequently, equating the two expressions for $\Delta W$, i.e.,

$$
\frac{F^{2} \Delta S}{2 S^{2}}=-2 t \int_{0}^{a} G(a) d a,
$$

we deduce the change of Young's modulus due to the presence of the crack of half-length $a$ (subscript a) compared to its theoretical (subscript th, i.e, defect-free) value in the following simple form

$$
\frac{E_{a}}{E_{t h}}=1-\frac{a^{2}}{r l} .
$$

Next we assume the presence of an additional transversal crack of half-length $b$, not interacting with the previous one. According to our previous result

$$
\frac{E_{a \oplus b}}{E_{a}}=1-\frac{E_{b}}{E_{t h}} \equiv \frac{E_{b}}{E_{t h}},
$$

where $E_{a \oplus b} \equiv E_{b \oplus a}$ denotes Young's modulus of the nanotube containing the two non interacting transversal cracks. Thus, we derive

$$
\frac{E_{a \oplus b}}{E_{t h}}=\frac{E_{a}}{E_{t h}} \frac{E_{b}}{E_{t h}}=\left(1-\frac{a^{2}}{r l}\right)\left(1-\frac{b^{2}}{r l}\right) .
$$

For interacting cracks the previous approach remains valid if $K=\sigma \sqrt{\pi a}$ is substituted with the corresponding value of the stress-intensity factor at the tips of two interacting cracks, which can be found in proper Handbooks. However, to have an idea of the possible role of the interaction we note that it will be maximal for collinear coalescing cracks. Thus at the coalescence,

$$
\frac{E_{a+b}}{E_{t h}}=1-\frac{(a+b)^{2}}{r l}
$$

and the maximum interaction is predicted to be

$$
\frac{E_{a \oplus b}-A_{a+b}}{E_{t h}}=\frac{a^{2} b^{2}+2 a b r l}{r^{2} l^{2}} \approx \frac{2 a b}{r l},
$$

where the last approximation is valid only for small crack lengths (with respect to $r$ and $l$ ).

We are now ready to derive a general law. Let us consider $N$ cracks having the size $a_{i}$ or, and that is the same, $M$ different cracks with multiplicity $N_{i}$ $\left(N=\sum_{i=1}^{M} N_{i}\right)$. Let $n_{i}=2 a_{i} / q$ represent the number of adjacent vacancies in the crack of half-length $a_{i}, q$ be the atomic size, and $f_{i}=\left(N_{i} n_{i}\right) /\left(2 \pi r l / q^{2}\right)$ be its related numerical vacancy fraction. Then we can write (the approximations are valid for small cracks)

$$
\begin{gathered}
\frac{E}{E_{t h}}=\prod_{i=1}^{N} \frac{E_{a_{i}}}{E_{t h}}=\prod_{i=1}^{N}\left(1-\frac{a_{i}^{2}}{r l}\right)=\prod_{i=1}^{M}\left(1-\frac{a_{i}^{2}}{r l}\right)^{N_{i}} \\
\approx 1-\sum_{i=1}^{M} \frac{N_{i} a_{i}^{2}}{r l}=1-\frac{\pi}{2} \sum_{i=1}^{M} f_{i} n_{i} .
\end{gathered}
$$

We note that our treatment can be viewed as a generalization of the approach proposed in [18], being able to quantify the constants $k_{i}$ fitted by atomistic simulations in ref. [18] for three different types of defects. In particular, rearranging eq. (1) and in the limit of three small cracks, we deduce

$$
\frac{E_{t h}}{E} \approx 1+k_{1} c_{1}+k_{2} c_{2}+k_{3} c_{3},
$$

which is identical to eq. (15) in [18], in which $c_{i}=N_{i} / l$ is the linear defect concentration and $k_{i}=n_{i}^{2} q^{2} /(4 r)$. The authors of [18] consider 1 , 2 and 3 atoms missing, with and without reconstructed bonds. For not reconstructed bonds two alternative defect orientations were investigated for 2 and 3 atoms missing (for details see [18]). Even 
Table 1: Comparison between present approach (bold numbers) and atomistic simulations, for defects with reconstructed and not reconstructed (italic numbers) vacancies (with different orientations for 2 and 3 atoms missing, see [18] for details). All the reported quantities are in Ångström (for a $(m, p)$ carbon nanotubes we simply consider here $q \approx 0.246 \mathrm{~nm}$, see [19], and $\left.r \approx 0.0392 \sqrt{m^{2}+p^{2}+m p} \mathrm{~nm}\right)$.

\begin{tabular}{|c|c|c|c|c|}
\hline$(m, p)$ & $r$ & $k_{1}\left(n_{1}=1\right)$ & $k_{2}\left(n_{2}=2\right)$ & $k_{3}\left(n_{3}=3\right)$ \\
\hline$(5,5)$ & 3.39 & $\mathbf{0 . 4 5} \quad 1.2 \quad 1.2$ & $\mathbf{1 . 7 9} 1.41 .72 .8$ & 4.021 .82 .23 .6 \\
\hline$(9,0)$ & 3.53 & 0.431 .11 .1 & $\begin{array}{lllll}1.71 & 1.2 & 1.3 & 2.1\end{array}$ & $3.861 .6 \quad 2.43 .6$ \\
\hline$(10,10)$ & 6.79 & 0.220 .80 .5 & $\mathbf{0 . 8 9} 1.00 .741 .3$ & $2.011 .2 \quad 1.01 .5$ \\
\hline$\overline{(17,0)}$ & 6.67 & $\begin{array}{llll}0.23 & 0.8 & 0.5\end{array}$ & $\begin{array}{lllll}0.91 & 1.0 & 0.7 & 1.0\end{array}$ & $\begin{array}{lllll}2.04 & 1.2 & 1.2 & 1.7\end{array}$ \\
\hline
\end{tabular}

if their defect geometries are much more complex than our considered nanocrack, the comparison between our approach and their atomistic simulations shows good agreement, as summarized in Table 1 .

Thus, if only one type of crack, formed by $n$ adjacent vacancies, is present with fraction $f$ in a space elevator cable, its Young's modulus $E(f, n)$ must satisfy:

$$
\frac{E(f, n)}{E_{t h}} \approx 1-\frac{\pi}{2} f n
$$

\subsection{Strength}

Contrary, the strength is influenced only by the most critical defect (i.e., is not a function of $f$ ); applying quantized fracture mechanics [19] $\left(G^{\star}=\right.$ $\left.-\Delta W / \Delta A=G_{C}\right)$ for the crack propagation we have found for the failure stress $\sigma_{f}^{(N T)}$ of a single nanotube

$$
\frac{\sigma_{f}^{(N T)}}{\sigma_{t h}} \approx \frac{1}{\sqrt{1+n}} .
$$

Thus, by imposing the longitudinal equilibrium of the entire cable, we derive its strength $\sigma_{f}(\nu, n)$ according to

$$
\frac{\sigma_{f}(\nu, n)}{\sigma_{t h}} \approx 1-\nu\left(1-\frac{1}{\sqrt{1+n}}\right),
$$

in which $\nu$ represents the fraction of defective nanotubes in the cable (we expect $\nu \approx 1$ ). Thus, small defects can strongly reduce the strength of a space elevator cable, as emphasized in a recent paper [20, whereas large damage is needed to have a strong stiffness reduction.

Some data for high strength steel, Boron fibre, Carbon fibre, Kevlar and Carbon nanotubes, is given in Table 2. For the Carbon nanotubes we present
(1) the theoretically calculated values, (2) the experimental values and (3) and (4) two sets of values calculated from eq. 3 and 4 . The difference in these values follows from assumptions concerning the defect concentration, which has a strong influence on the stiffness. The detailed calculations are explained in [18].

\section{Reduced Energy Momentum Method (REMM)}

The REMM is the proper mathematical method to investigate the stability of relative equilibria in symmetric Hamiltonian systems. Mathematically speaking, a relative equilibrium is a solution, whose orbit coincides with an one parameter group orbit of the symmetry group of the system. For the problem treated in this paper the symmetry group is the planar rotation group. In engineering language a relative equilibrium is an equilibrium in a properly moving (in this case rotating) coordinate frame. Whereas for nonsymmetric Hamiltonian systems the stability test according to LagrangeDirichlet requires for an equilibrium to be stable that the second variation of the potential $V(\boldsymbol{q})$ must be positive definite, in the symmetric case the situation is more complicated. Now the invariance against the symmetry group motion (rotation) and consequently the existence of additional conserved quantities must be included in the analysis by forming the socalled amended potential $V_{\mu_{0}}$ defined by

$$
V_{\boldsymbol{\mu}_{0}}(\boldsymbol{q})=V(\boldsymbol{q})+\frac{1}{2} \boldsymbol{\mu}_{0} \cdot \mathcal{J}^{-1}(\boldsymbol{q}) \boldsymbol{\mu}_{0} .
$$

Here $\mathcal{J}(\boldsymbol{q})$ is the "locked inertia tensor", which is a generalization of the inertia tensor of the rigid 
Table 2: Data for some high strength materials. $\sigma_{c}$ : tensile strength; E: Youngs modulus; $\varrho$ : density.

\begin{tabular}{|l|l|c|c|c|c|}
\hline Material & Abbr. & $\sigma_{c}[\mathrm{GPa}]$ & $E[\mathrm{GPa}]$ & $\varrho\left[\mathrm{kg} / \mathrm{m}^{3}\right]$ & $\epsilon_{c}$ \\
\hline Steel & St & $1-5$ & 200 & 7900 & 0.025 \\
Boron fiber & & 3.5 & 400 & 2450 & 0.087 \\
Kevlar & & 3.6 & 127 & 1440 & 0.028 \\
Carbon fiber & $\mathrm{CF}$ & $2-5$ & $250-830$ & 1850 & 0.008 \\
Carbon nanotubes (theoretical data)(1) & CTT & 150 & 630 & 1300 & 0.238 \\
Carbon nanotubes (experimental data)(2) & CTE & 65 & 630 & 1300 & 0.103 \\
Carbon nanotubes (according to (3.,4)(3) & CTT0.6 & 65 & 378 & 1300 & 0.516 \\
Carbon nanotubes (according to (3), 4)(4) & CTT0.2 & 65 & 126 & 1300 & 0.172 \\
\hline
\end{tabular}

body motion obtained by locking the deformability in the configuration $\boldsymbol{q}$ obtained from the group motion. The conserved quantity $\boldsymbol{\mu}_{0}$ is the angular momentum for the relative equilibrium configuration.

In order to avoid taking the second derivative of the inverse of the locked inertia tensor in (5) for the calculation of the second variation of $V_{\mu_{0}}$, it is convenient to make use of the expression

$$
\begin{aligned}
\mathbf{D}^{2} V_{\mu_{0}}\left(\boldsymbol{q}_{0}\right)(\boldsymbol{\delta} \boldsymbol{q}, \boldsymbol{\delta} \boldsymbol{q})=\mathbf{D}^{2} V_{\xi_{0}}\left(\boldsymbol{q}_{0}\right)(\boldsymbol{\delta} \boldsymbol{q}, \boldsymbol{\delta} \boldsymbol{q}) \\
+\operatorname{ident}_{\xi_{0}}(\boldsymbol{\delta} \boldsymbol{q}) \cdot \mathcal{J}^{-1}\left(\boldsymbol{q}_{0}\right) \operatorname{ident}_{\xi_{0}}(\boldsymbol{\delta} \boldsymbol{q})
\end{aligned}
$$

where

$$
\operatorname{ident}_{\xi_{0}}(\boldsymbol{\delta} \boldsymbol{q}):=-\left(\mathbf{D} \mathcal{J}\left(\boldsymbol{q}_{0}\right) \cdot \boldsymbol{\delta} \boldsymbol{q}\right) \cdot \boldsymbol{\xi}_{0},
$$

and

$$
V_{\xi}(\boldsymbol{q})=V(\boldsymbol{q})-\frac{1}{2} \boldsymbol{\xi} \cdot \mathcal{J}(\boldsymbol{q}) \boldsymbol{\xi}
$$

is the augmented potential. $\boldsymbol{\xi}$ is the angular velocity. The subscript 0 indicates that the quantities in (7) are evaluated at the relative equilibrium.

For a relative equilibrium to be stable the second variation given by (6) must be positive definite.

\section{Simple model: Dumbell- Satellite}

In order to show the necessary steps we consider first the dumbell-satellite of Fig. 3, treated in Krupa et al. [8]. It consists of two point masses connected by a massless rigid rod of length $2 a$.

The expressions necessary to evaluate the stability condition of the radial relative equilibrium for

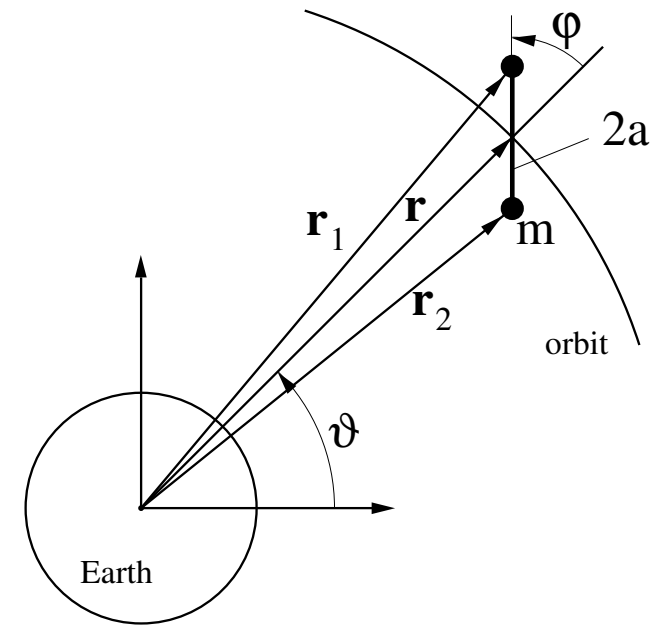

Figure 3: Planar motion of a dumbell-satellite of length $2 a$ on a circular orbit around the Earth

the dumbell satellite are the kinetic and potential energies:

$$
\begin{aligned}
T= & m\left(\dot{r}^{2}+r^{2} \dot{\vartheta}^{2}+a^{2}(\dot{\vartheta}+\dot{\varphi})^{2}\right), \\
V= & -\frac{k m}{\sqrt{r^{2}+2 r a \cos \varphi+a^{2}}} \\
& -\frac{k m}{\sqrt{r^{2}-2 r a \cos \varphi+a^{2}}},
\end{aligned}
$$

where $k$ is the gravitational constant of the Earth. The locked inertia tensor is given by (Krupa et al. [8])

$$
\mathcal{J}(\boldsymbol{q}) \cdot \xi=J\left(\boldsymbol{q}, F L\left(\xi_{Q}(\boldsymbol{q})\right)\right)=2 m\left(r^{2}+a^{2}\right) \xi
$$

The augmented potential (8), expressed by the angular velocity $\dot{\vartheta}=\xi$ reads

$$
V_{\xi}=V-m\left(r^{2}+a^{2}\right) \xi^{2}
$$


and the amended potential (5) expressed by angular momentum $\mu_{0}=\mathcal{J} \xi$ is given by

$$
V_{\mu_{0}}=V+\frac{\mu_{0}^{2}}{4 m\left(r^{2}+a^{2}\right)} .
$$

The equilibrium position is stable, if $V_{\mu_{0}}^{\prime \prime}$ is positive definite. For the radial relative equilibrium position we obtain as deciding quantity ([8])

$$
\frac{\partial^{2} V_{\mu}}{\partial r^{2}}=\frac{2\left((r / a)^{4}-10(r / a)^{2}+1\right)}{\left((r / a)^{2}-1\right)^{3}}>0
$$

For $r / a<\sqrt{5+2 \sqrt{6}} \approx 3.14626$ the second derivative $\partial^{2} V_{\mu} / \partial r^{2}$ becomes negative. This means that fixing the radius $r$ of the orbit and increasing the distance $a$ between the two masses beyond the given value, results in the instability of the radial relative equilibrium position.

From this example we draw the following conclusions, which will be used to simplify the following analysis of the continuous problem:

1. Only the planar problem has to be considered. This is justified by the analysis given in Krupa et al. [8] and Krupa et al. [16], where it is shown that the perturbation out of the orbital plane completely decouples and is always stable.

2. Moreover, it follows both from the general theory of the REMM, where a block diagonalization of the second derivative is predicted, and our calculations, that also the stability analysis of the angular motion (attitude motion) of the string in the orbital plane and of the radial motion decouple and hence, as it is shown above, only the radial variation results in the relevant stability condition.

\section{Continuous string model}

Following the conclusions drawn at the end of the preceeding Section for the treatment of the dumbell satellite we simplify the stability problem. Due to the block diagonalization of the second variation we only perform the second variation with respect to the radial coordinate. To give a general proof that the perturbation concerning the attitude motion is always stable, one has to prove that the inequality

$$
\begin{aligned}
0 \leq & \int_{s_{0}}^{s_{1}}\left\{E A(s) \frac{r^{\prime}-1}{r^{\prime}}\left(\delta y^{\prime}\right)^{2}\right. \\
& \left.+\varrho A(s)\left(\frac{k}{r^{2}}-\xi^{2} r\right)(\delta y)^{2}\right\} d s \\
& +m_{0}\left(\frac{k}{r_{0}^{2}}-\xi^{2} r_{0}\right)(\delta y)^{2}\left(s_{0}\right) \\
& +m_{1}\left(\frac{k}{r_{1}^{2}}-\xi^{2} r_{1}\right)(\delta y)^{2}\left(s_{1}\right) \\
& +m_{s}\left(\frac{k}{r_{g s}^{2}}-\xi^{2} r_{g s}\right)(\delta y)^{2}\left(s_{g s}\right)
\end{aligned}
$$

holds for all nontrivial transversal variations $\delta y(s)$ satisfying the integral constraint

$$
\begin{aligned}
0 & =\int_{s_{0}}^{s_{1}} \varrho A(s) r(s) \delta y(s) d s+m_{0} r\left(s_{0}\right) \delta y\left(s_{0}\right) \\
& +m_{1} r\left(s_{1}\right) \delta y\left(s_{1}\right)+m_{s} r\left(s_{g s}\right) \delta y\left(s_{g s}\right) .
\end{aligned}
$$

Obviously the last term in (10) vanishes, because the gravitational and centrifugal accelerations cancel out at the geostationary orbit. The integral constraint (11) follows from the definition of the internal vibration modes given by the REMM. We note that (11) excludes rigid rotations around the center of the Earth because for a rotation $y(s) \propto r(s)$ the right hand side in (10) vanishes. Unfortunately we were not yet able to show analytically that 10 ) holds for all admissible transversal variations, because the coefficient of $(\delta y)^{2}$ is negative outside the geo-stationary orbit. However, all numerical calculations carried out by discretising the massive string resulted in stability concerning the attitude motion. Hence we proceed as indicated, checking only the radial variation of the amended potential.

We consider now a system consisting of masses $m_{0}$ at the inner radius $r_{0}, m_{1}$ at the outer radius $r_{1}$, and the satellite (space station) with $m_{s}$ at the geostationary orbit $r_{g s}=r\left(s_{g s}\right)$, connected by a linearly elastic massive (density $\rho$ ) string. Analogously as in the preceeding Section we need, according to (5), the quantities $V, T$ and $\mathcal{J}$, which are given by

$$
\begin{aligned}
V= & \int_{s_{0}}^{s_{1}}\left(\frac{E A}{2}\left(r^{\prime}-1\right)^{2}-\frac{k \varrho A}{r}\right) d s \\
& -\frac{m_{0} k}{r\left(s_{0}\right)}-\frac{m_{1} k}{r\left(s_{1}\right)}-\frac{m_{s} k}{r\left(s_{g s}\right)},
\end{aligned}
$$


$T=\frac{\mathcal{J}}{2} \xi^{2}=\frac{\mu_{0}^{2}}{2 \mathcal{J}}$

$\mathcal{J}=\int_{s_{0}}^{s_{1}} \varrho A r^{2} d s+m_{0} r\left(s_{0}\right)^{2}+m_{1} r\left(s_{1}\right)^{2}+m_{s} r\left(s_{g s}\right)^{2}$.

Inserting the first variations

$$
\begin{aligned}
\delta V= & \int_{s_{0}}^{s_{1}}\left(E A\left(r^{\prime}-1\right) \delta r^{\prime}+\frac{k \varrho A}{r^{2}} \delta r\right) d s \\
& +\frac{m_{0} k}{r^{2}\left(s_{0}\right)} \delta r\left(s_{0}\right)+\frac{m_{1} k}{r^{2}\left(s_{1}\right)} \delta r\left(s_{1}\right) \\
& +\frac{m_{s} k}{r^{2}\left(s_{g s}\right)} \delta r\left(s_{g s}\right) \\
\delta T= & -\frac{\mu^{2}}{2 \mathcal{J}^{2}} \delta \mathcal{J}=-\frac{\xi^{2}}{2} \delta \mathcal{J} \\
\delta \mathcal{J}= & \int_{s_{0}}^{s_{1}} 2 \varrho A r \delta r d s+2 m_{0} r\left(s_{0}\right) \delta r\left(s_{0}\right) \\
& +2 m_{1} r\left(s_{1}\right) \delta r\left(s_{1}\right)+2 m_{s} r\left(s_{g s}\right) \delta r\left(s_{g s}\right) .
\end{aligned}
$$

into

$$
\delta V+\delta T=0
$$

and performing integration by parts to remove $\delta r^{\prime}$ results in one field equation

$$
E\left(A\left(r^{\prime}-1\right)\right)^{\prime}=\frac{k \varrho A}{r^{2}}-\xi^{2} \varrho A r,
$$

with the boundary and switching conditions

$$
\begin{aligned}
\left.E A\left(r^{\prime}-1\right)\right|_{s_{0}} & =\frac{k m_{0}}{r^{2}\left(s_{0}\right)}-\xi^{2} m_{0} r\left(s_{0}\right), \\
\left.E A\left(r^{\prime}-1\right)\right|_{s_{1}} & =-\frac{k m_{1}}{r^{2}\left(s_{1}\right)}+\xi^{2} m_{1} r\left(s_{1}\right), \\
\left.E A\left(r^{\prime}-1\right)\right|_{s_{g s}^{-}} ^{s_{g s}^{+}} & =\frac{k m_{s}}{r^{2}\left(s_{g s}\right)}-\xi^{2} m_{s} r\left(s_{g s}\right)
\end{aligned}
$$

From these equations the tension $\sigma(s)$ in the string and the extension of the string can be calculated. Since the right hand side in the jump condition (14.) vanishes, the normal force $E A\left(r^{\prime}-1\right)$ remains continuous at the space station and the jump condition can be neglected in the computation of the equilibrium configuration. In order to have a minimum weight design, the cross section $A(s)$ of the string is varied such that the tension $\sigma$ reaches its maximum admissible value in each cross section, that is,

$$
\sigma=E\left(r^{\prime}-1\right) \equiv \sigma_{c} .
$$

From this relation follows

$$
r^{\prime}-1=\sigma_{c} / E
$$

Inserting 150 into $(13)$ results in

$$
A^{\prime} / A=\frac{\varrho}{\sigma_{c}}\left(k / r^{2}-\xi^{2} r\right) .
$$

From (16) the shape of the string between the two end masses can be calculated. It is shown in Fig. 4 for carbon nanotubes with the four different values of $\sigma_{c}$ given in Table 2 and endmasses of $1 \mathrm{~kg}$ each.

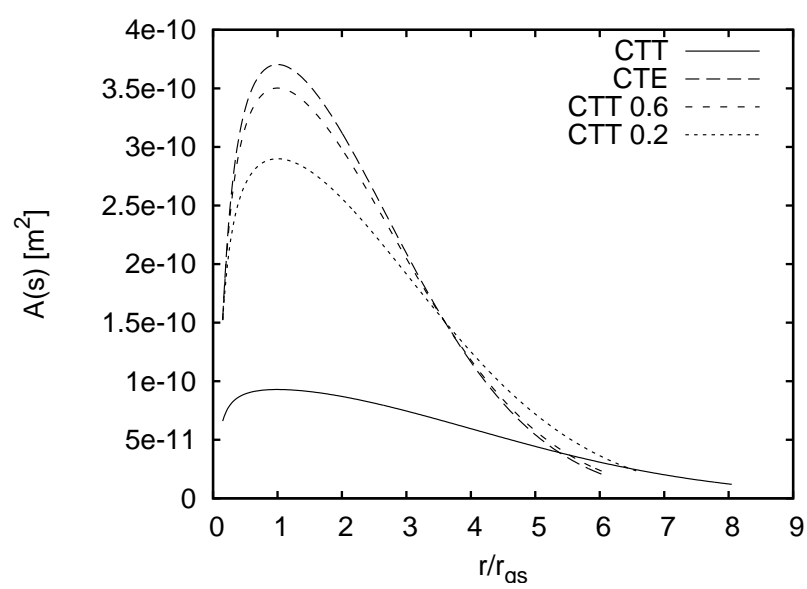

Figure 4: Cross-sectional areas of the four carbon nanotube strings from Table 2 and end masses of $1 \mathrm{~kg}$.

The string with the higher admissible stress has a cross-sectional area of $6.6 \cdot 10^{-11} \mathrm{~m}^{2}$ at the surface of the Earth. At the geostationary height the crosssectional area is $9.3 \cdot 10^{-11} \mathrm{~m}^{2}$, thus giving the ratio 1.41, which is a little bit smaller than the ratio given in Edwards [5]. The taper ratios for the cases numbered in Table 2 are listed in Table 3.

Table 3: Taper ratios for the cases in Table 2,

\begin{tabular}{|l|c|c|c|}
\hline Case & Taper ratio & $A\left(r_{0}\right)\left[\mathrm{m}^{2}\right]$ & $A\left(r_{\mathrm{gs}}\right)\left[\mathrm{m}^{2}\right]$ \\
\hline CTT & 1.41 & $6.6 \mathrm{e}-11$ & $9.3 \mathrm{e}-11$ \\
CTE & 2.43 & $1.5 \mathrm{e}-10$ & $3.7 \mathrm{e}-10$ \\
CTT0.6 & 2.30 & $1.5 \mathrm{e}-10$ & $3.5 \mathrm{e}-10$ \\
CTT0.2 & 1.90 & $1.5 \mathrm{e}-10$ & $2.9 \mathrm{e}-10$ \\
\hline
\end{tabular}

\section{Determination of orbital sta- bility of the radial relative equilibrium position}

To guarantee stability of the radial relative equilibrium position it is necessary and sufficient that the 
second variation of (5) is positive definite (Krupa et al. [16]). Inserting

$$
\begin{aligned}
\delta^{2} V= & \int_{s_{0}}^{s_{1}}\left(E A\left(\delta r^{\prime}\right)^{2}-\frac{2 k \varrho A}{r^{3}}(\delta r)^{2}\right) d s \\
& -\frac{2 m_{0} k}{r^{3}\left(s_{0}\right)}\left(\delta r\left(s_{0}\right)\right)^{2}-\frac{2 m_{1} k}{r^{3}\left(s_{1}\right)}\left(\delta r\left(s_{1}\right)\right)^{2} \\
& -\frac{2 m_{2} k}{r^{3}\left(s_{g s}\right)}\left(\delta r\left(s_{g s}\right)\right)^{2} \\
\delta^{2} T= & \frac{\mu^{2}}{\mathcal{J}^{3}}(\delta \mathcal{J})^{2}-\frac{\mu^{2}}{2 \mathcal{J}^{2}} \delta^{2} \mathcal{J} \\
\delta \mathcal{J}= & \int_{s_{0}}^{s_{1}} 2 \varrho A r \delta r d s+2 m_{0} r\left(s_{0}\right) \delta r\left(s_{0}\right) \\
& +2 m_{1} r\left(s_{1}\right) \delta r\left(s_{1}\right)+2 m_{s} r\left(s_{g s}\right) \delta r\left(s_{g s}\right), \\
\delta^{2} \mathcal{J}= & \int_{s_{0}}^{s_{1}} 2 \varrho A(\delta r)^{2} d s+2 m_{0}\left(\delta r\left(s_{0}\right)\right)^{2} \\
& +2 m_{1}\left(\delta r\left(s_{1}\right)\right)^{2}+2 m_{s}\left(\delta r\left(s_{g s}\right)\right)^{2} .
\end{aligned}
$$

into $\delta^{2} V+\delta^{2} T$, we have to check the positive definiteness. It is explained in Krupa et al. [16] that this can be done at least in two different ways. We take the approach, where a minimization problem is formulated, because this results in an accurate determination of the smallest eigenvalue, the sign of which determines the stability of the configuration (Krupa et al. [16]).

Hence we formulate the quadratic minimization problem

$$
\min _{\|\delta r\|_{w}^{2}=1}\left(\delta^{2} V+\delta^{2} T\right)\left(\delta r, \delta r^{\prime}\right)
$$

where

$$
\begin{aligned}
\|\delta r\|_{w}^{2}= & \int_{s_{0}}^{s_{1}} \rho A \delta r^{2}(s) d s+m_{0}\left(\delta r\left(s_{0}\right)\right)^{2} \\
& +m_{1}\left(\delta r\left(s_{1}\right)\right)^{2}+m_{s}\left(\delta r\left(s_{g s}\right)\right)^{2} .
\end{aligned}
$$

As outlined in [21, we reformulate this isoperimetric minimization problem as Optimal Control problem, because this formulation gives sligthly simpler equations than the analogous Lagrangian formulation. After some calculations we obtain the boundary value problem

$$
\begin{aligned}
\delta r^{\prime} & =\delta N /(E A) \\
\delta N^{\prime} & =\left(\frac{-2 k}{r^{3}}-\xi^{2}-\lambda\right) \varrho A \delta r \\
& +2 \varrho A \xi^{2} r \delta \xi \\
s=s_{0}: \delta N & =m_{0}\left(\frac{-2 k}{r^{3}}-\xi^{2}-\lambda\right) \delta r
\end{aligned}
$$

$$
\begin{aligned}
& +2 m_{0} \xi^{2} r \delta \xi \\
s=s_{1}: \delta N & =-m_{1}\left(\frac{-2 k}{r^{3}}-\xi^{2}-\lambda\right) \delta r \\
& -2 m_{1} \xi^{2} r \delta \xi \\
s=s_{g s}: \delta N\left(s_{g s}^{+}\right) & =\delta N\left(s_{g s}^{-}\right) \\
& +m_{s}\left(\frac{-2 k}{r^{3}}-\xi^{2}-\lambda\right) \delta r \\
& +2 m_{s} \xi^{2} r \delta \xi,
\end{aligned}
$$

with the scaling condition

$$
1=\|\delta r\|_{w}^{2} .
$$

$\delta N$ denotes the virtual axial force in the string. Positive eigenvalues $\lambda$ assure stability.

Comparing (18) with (13) and (14), we find that (18) is just the eigenvalue problem for the linearization of the BVP (13) and (14).

\section{$7 \quad$ Numerical results}

We consider first the string without a satellite at the geostationary height $\left(m_{s}=0\right)$. In Fig. 5 the smallest eigenvalue of (6) is depicted against the ratio $r_{0} / r_{g s}$. If this ratio is equal to 1 , the string is of zero length and if it is equal to 0.151 , the inner radius $r_{0}$ is equal to the radius of the Earth. Hence in this case the string extending down from the geostationary height touches the surface of the Earth. We can see from Fig. 5, that for short string lengths the radial relative equilibrium of a string moving with geostationary angular velocity is stable for all materials, since $\lambda$ is positive. Increasing the length of the string, $\lambda$ becomes negative for all strings made from nanotubes, when the inner radius $r_{0}$ decreases to approx. $0.8 r_{g s}$.

Up to now we neglected the influence of the space station's mass $\left(m_{s}=0\right)$ on the orbital stability. Since the geostationary orbit of the space station, without strings attached to it, is orbitally stable, a sufficiently heavy space station should be able to stabilize the configuration.

We proceed now in the following way: Keeping the eigenvalue $\lambda \equiv 0$ and regarding $m_{s}$ as additional unknown variable, we calculate the necessary mass $m_{s}$ of the space station, for increasing string length, to stabilize the radial configuration of the system. In Fig.6, which is related to Fig. 5, we depict $m_{s}$ 


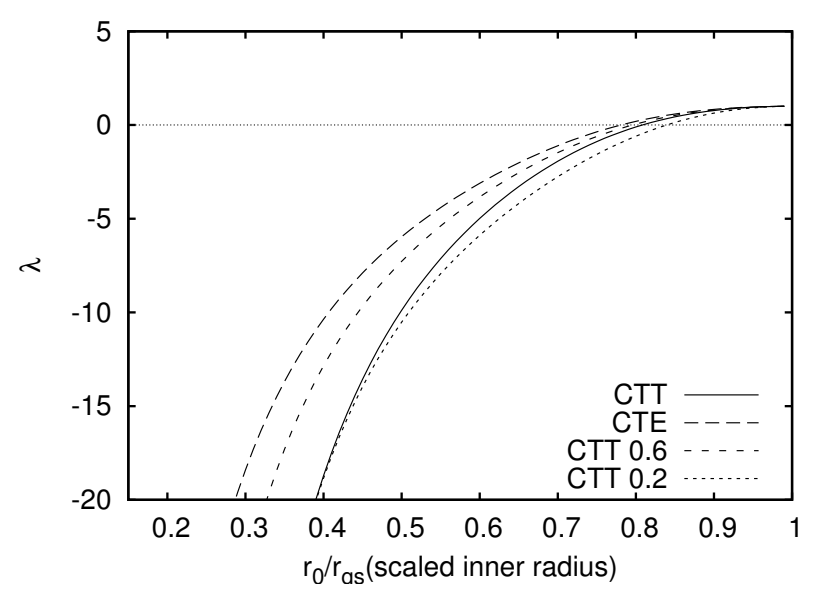

Figure 5: Smallest eigenvalue $\lambda$ for the string without intermediate satellite $\left(m_{s}=0\right)$ depicted against the ratio $r_{0} / r_{g s}$ for four different materials. Only for length ratios, where $\lambda$ is positive, the second variation of (6) is positive definite. For abbreviations in the Figure see Table 2.

for all four strings made from carbon nanotubes for the values Table 2, At the length, where the radial string configuration without intermediate satellite looses stability, the mass necessary for stabilisation becomes nonzero and grows for increasing string length. Three of the four strings are too soft and consequently no radial equilibrium configuration exists, no matter how big the mass of the space station is selected. The reason for this nonexistence of an equilibrium is explained with a simpler example in the Appendix.

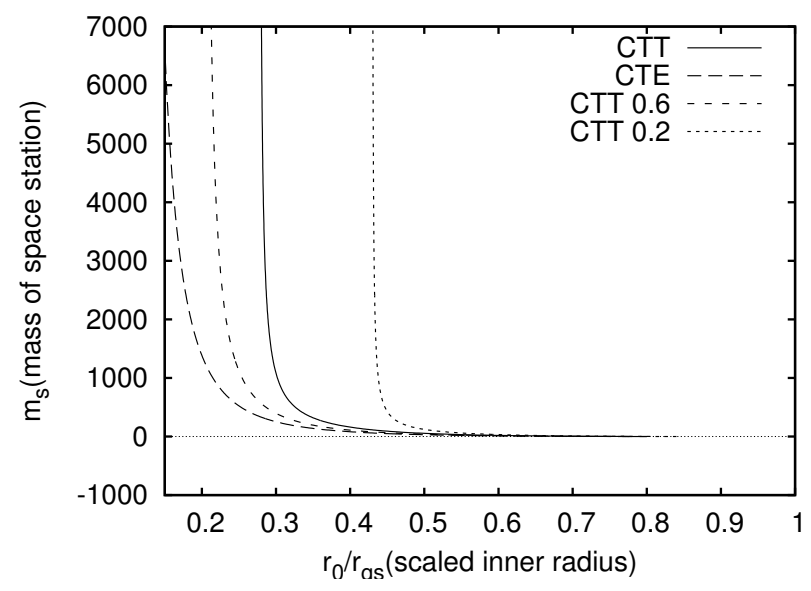

Figure 6: Minimum mass $m_{s}([\mathrm{~kg}])$ of the space station necessary to stabilize the radial relative equilibrium for the four strings of Table 1.

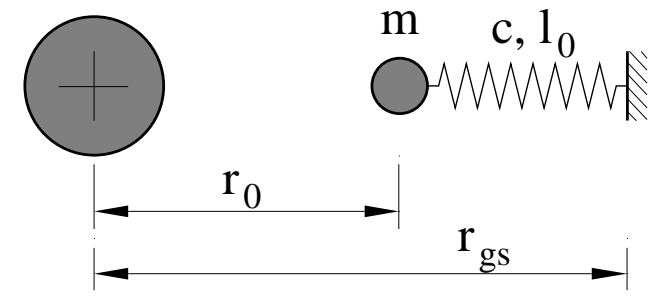

Figure 7: Spring pendulum in its radial relative equilibrium position in geostationary orbit

\section{Conclusions}

The main result of our investigation is that the radial relative equilibrium of a tapered string made from carbon nanotubes moving on a circular geostationary orbit and reaching from the surface of the Earth into space is orbitally unstable. However, its configuration can be stabilized by attaching to it a sufficiently heavy satellite in geostationary height. We calculated the minimum mass necessary for this satellite to achieve stabilization. Moreover, we also obtained the practically important result that for too soft strings the stabilization of the orbitally unstable relative equilibrium is not possible, because due to the large extension no relative equilibrium exists.

\section{Appendix: Spring pendulum in geostationary orbit}

We consider the simple spring pendulum sketched in Fig. 7. If it moves on a circular orbit in its radial relative equilibrium position with geostationary angular velocity, the equilibrium equation reads

$$
c\left(r_{g s}-r_{0}-l_{0}\right)=\frac{k m}{r_{0}^{2}}-m r_{0} \omega^{2} .
$$

Inserting the angular velocity of the geostationary orbit

$$
\omega^{2}=\frac{k}{r_{g s}^{3}}
$$

we obtain

$$
\frac{c}{k m}\left(r_{g s}-r_{0}-l_{0}\right)+\frac{r_{0}}{r_{g s}^{3}}=\frac{1}{r_{0}^{2}},
$$

which we rewrite as

$$
\gamma\left(a-b r_{0}\right)=\frac{1}{r_{0}^{2}}
$$


where

$$
\gamma=\frac{c}{k m}, \quad a=r_{g s}-l_{0} \quad \text { and } \quad b=1-\frac{1}{\gamma r_{g s}^{3}} .
$$

We introduce the scaling $r_{0}=\alpha r$ into 19 to obtain

$$
\alpha^{2} \gamma a-\alpha^{3} b \gamma r=\frac{1}{r^{2}} .
$$

Setting

$$
\alpha^{2} \gamma a=1 \quad \text { or } \quad \alpha=\frac{1}{\sqrt{a \gamma}}
$$

we obtain from 20

$$
1-\beta r=\frac{1}{r^{2}}
$$

where

$$
\beta=\frac{\alpha b}{a} .
$$

Equation (21) has a solution only if $\beta$ is small enough, because if $\beta$ is too large, there is no intersection between the straight line and the hyperbola.

If we resubstitute the physical quantities, it follows that the stiffness $c$ must be large enough, meaning that the extension is below a certain limit.

This agrees with the result, which was obtained for the continuous case.

\section{Acknowledgement}

Financial support of this work was given by the Austrian Science Foundation (FWF) and by INTAS.

\section{References}

[1] Artsutanov, Yu., 'Into Space on an Electric Locomotive', Komsomolskaya Pravda, 31 July 1960.

[2] Isaacs, J. D. Vine, A. C., and Bradner, H., 'Satellite Elongation into a true Sky-Hook', Sience, 151, 1966, 682-683.

[3] Pearson, J., 'The orbital tower: a spacecraft launcher using the Earth's rotational energy', Acta Astronautica, 2, 1975, 785-799.
[4] Beletsky, V.V., Levin, E. M., 'Dynamics of Space Tether Systems', Advances of the Astronautical Sciences, 83, 1993.

[5] Edwards B.C., 'Design and Deployment of a Space Elevator', Acta Astronautica, 47, 2000, 735-744.

[6] Ruoff, R. S., Quian, D., Liu, W. K. 'Mechanical properties of carbon nanotubes: Theoretical predictions and experimental measurements', C.R.Physique 4 (2003) 993-1008.

[7] Pantano, A., Parks, D. M., Boyce, M. C. 'Mechanics of deformation of single- and multiwall carbon nanotubes', J. of the Mechanics and Physics of Solids, 52, 2004, 789-821.

[8] Krupa, M., Steindl A., Troger, H., 'Stability of Relative Equilibria. Part II: Dumbell Satellites', Meccanica 35:353-371, 2001.

[9] Steindl, A., and Troger, H., 'Center Manifold Approach to the Control of a Tethered Satellite System', Applied Mathematics and Computation, 70, 1995, 315-327.

[10] Simo, J. C., Lewis, D.,'Energy methods in the stability analysis of relative equilibria of Hamiltonian systems'. The Proceedings of the sixth Symposium on Continuum Models and Discrete Systems, Dijon, June 1989, 162183. Editor: G.A. Maugin. Longman.

[11] Marsden, J. E., O'Reilly, O. M., Wicklin, F. J. and Zombro, B. W., Symmetry, Stability, Geometric Phases, and Mechanical Integrators (Part I), Nonlinear Science Today, vol.1, No. 1, 4-11, 1991.

[12] Marsden, J.E., Lectures on Mechanics, London Math. Society, Lect. Note Series 174, Cambridge University Press, 1992.

[13] Marsden, J.E. and Ratiu, T.S., An Introduction to Mechanics and Symmetry, A Basic Exposition of Classical Mechanical Systems, Springer-Verlag, New York - Heidelberg - Berlin, 1994.

[14] Karapetyan, A. V., Rumyantsev V.V., 'Stability of Conservative and Dissipative Systems', Applied Mechanics: Soviet Reviews, 
Volume 1: Stability and Analytical Mechanics, 1-145, 1983, Hemisphere Publishing

[15] Wang, L.-S., Chern, S.-J., Shih, C.-W., 'On the dynamics of a tethered satellite system', Archives for Rational Mechanics and Analysis, 127; 1994, 297-318.

[16] Krupa, M., Schagerl M., Steindl A., Szmolyan, P. and Troger, H., 'Relative equilibria of tethered satellite systems and their stability for very stiff tethers', DYNAMICAL SYSTEMS 16, 2001, pp. 253-287.

[17] A. Carpinteri, Structural mechanics: a unified approach. E \& FN SPON, (1997).

[18] M. Sammalkorpi, A. Krasheninnikov, A. Kuronen, K. Nordlund, K. Kaski, Mechanical properties of carbon nanotubes with vacancies and related defects, PHYSICAL REVIEW B, (2004) 70, 245416-1/8.

[19] N. Pugno, R. Ruoff, Quantized Fracture Mechanics, PHILOSOPHICAL MAGAZINE, (2004) 84/27, 2829-2845.

[20] N. Pugno, On the strength of the nanotubebased space elevator cable: from nanomechanics to megamechanics. J. OF PHYSICS

[21] Leitmann G., The Calculus of Variations and Optimal Control, Mathematical Concepts and Methods in Science and Engineering, vol. 24, Plenum Press, New York and London, 1981. 